\title{
The distribution of oxygen on the surface of the Ap star $\theta$ Aur
}

\section{An abundance Doppler image to compare with $\epsilon \mathrm{UMa}$}

\author{
J. B. Rice ${ }^{1}$, D. E. Holmgren ${ }^{2 \star \star}$, and D. A. Bohlender ${ }^{3}$ \\ 1 Department of Physics and Astronomy, Brandon University, Brandon, MB, R7A 6A9 Canada \\ e-mail: rice@brandonu.ca \\ 2 SMART Technologies Inc., Bay 8, 1460-26th Street NE, Calgary, AB T2A 7W6, Canada \\ e-mail: DavidHolmgren@smarttech.com \\ 3 Herzberg Institute of Astrophysics, National Research Council of Canada, 5071 West Saanich Road, Victoria, BC, \\ V9E 2E7, Canada \\ e-mail: david.bohlender@nrc-cnrc.gc.ca
}

Received 6 November 2003 / Accepted 23 May 2004

\begin{abstract}
We present a Doppler Image of the oxygen abundance distribution over the surface of the star $\theta$ Aur. The symmetry of the oxygen distribution appears to be consistent with the magnetic field observations such that the oxygen is highly depleted at the magnetic poles. In contrast to the star $\epsilon \mathrm{UMa}$, where the oxygen displayed undepleted solar abundance in a band around the magnetic equator, for $\theta$ Aur the instances of more normal solar levels of abundance occur in bands midway between the magnetic poles and the magnetic equator. The oxygen abundance at the magnetic equator is only mildly depleted for $\theta$ Aur. It is suggested that the oxygen abundance distribution is consistent with a global field that has a strong quadrupolar component. A detailed comparison is made between the oxygen abundance distribution and that of the distribution of chromium as mapped by Hatzes (1991a). A striking asymmetry is apparent in the contrasting behaviour of the oxygen and chromium abundance pattern at the two magnetic poles.
\end{abstract}

Key words. stars: chemically peculiar

\section{Introduction}

$\theta$ Aur (HD 40312) is a B9p star that was first discussed by Hiltner \& Morgan (1944) as a modestly chemically peculiar star with variable spectral line profiles. Two papers, van Rensbergen et al. (1984) and Adelman et al. (1984), constituted a detailed single large high resolution spectroscopic investigation of the star and its variability. Shortly before the publication of these papers, $\theta$ Aur had been included by Borra $\&$ Landstreet (1980) in a catalog of stars for which they had obtained magnetic field observations with their $\mathrm{H} \beta$ polarimeter. The effective $B_{\mathrm{e}}$ field variation was observed to be an almost sinusoidal waveform with very little scatter in the observations and the variation ranged between the extrema of $-240 \mathrm{G}$ and +360 G. Doppler images of the distribution of iron, chromium and silicon were reported by Khokhlova et al. (1986) and by Rice \& Wehlau $(1990,1991)$. A later paper by Hatzes (1991a) showed that $\theta$ Aur exhibited a distribution of chromium over the surface such that there was a depleted band of the element located at approximately the assumed location of the magnetic

* Guest Investigator, Dominion Astrophysical Observatory, Herzberg Institute of Astrophysics, National Research Council of Canada. equator. This distribution of chromium was similar to that of three stars he had reported on in an earlier paper, $\epsilon \mathrm{UMa}$, 45 Her, and $\omega$ Her (see Hatzes 1991b).

The motivation for this paper on the distribution of oxygen over the surface of $\theta$ Aur arose from earlier work on the unusual oxygen distribution on Ap stars that began with a paper by Gonzales \& Artru (1994). Their observations of the O I triplet at $7775 \AA$ in several Ap stars showed that these lines exhibit very dramatic line profile variations. Gonzalez \& Artru interpreted the line profile variations for one star, $\epsilon \mathrm{UMa}$, in terms of a belt of very enhanced abundance about the presumed magnetic equator of the star. It was clear that with a more complete set of data and detailed Doppler imaging, a very good map of the oxygen distribution could be obtained to complement existing iron and chromium abundance maps. Papers by Rice et al. (1997) and Holmgren \& Rice (2000) revealed sharply defined belts of oxygen and calcium at the magnetic equator. In particular, for oxygen the abundance pattern consists of a normal solar abundance in the well-defined equatorial belt, while at the magnetic poles oxygen is depleted by up to five orders of magnitude from solar abundances. In addition, secondary patches of oxygen are clearly evident that might suggest that the source of the non-uniform distribution of oxygen could be a 
Table 1. Observation $\log$ for $\theta$ Aur at $7775 \AA$.

\begin{tabular}{|c|c|c|c|}
\hline UT date & HJD (245000+) & Phase & $\overline{\text { Phase }\left({ }^{\circ}\right)}$ \\
\hline \multirow[t]{3}{*}{$2000-4-7$} & 1641.7356 & 0.589 & 212.0 \\
\hline & 1641.7837 & 0.602 & 216.7 \\
\hline & 1641.8150 & 0.611 & 220.0 \\
\hline \multirow[t]{4}{*}{$2000-4-8$} & 1642.6989 & 0.855 & 307.8 \\
\hline & 1642.7433 & 0.867 & 312.1 \\
\hline & 1642.7871 & 0.879 & 316.4 \\
\hline & 1642.8308 & 0.892 & 321.1 \\
\hline \multirow[t]{4}{*}{ 2000-4-9 } & 1643.7183 & 0.137 & 49.3 \\
\hline & 1643.7703 & 0.151 & 54.4 \\
\hline & 1643.8155 & 0.164 & 59.0 \\
\hline & 1643.8731 & 0.180 & 64.8 \\
\hline \multirow[t]{3}{*}{ 2000-4-10 } & 1644.7626 & 0.425 & 153.0 \\
\hline & 1644.7855 & 0.432 & 155.5 \\
\hline & 1644.8230 & 0.442 & 159.1 \\
\hline \multirow[t]{3}{*}{ 2000-4-11 } & 1645.7042 & 0.686 & 247.0 \\
\hline & 1645.7528 & 0.699 & 251.6 \\
\hline & 1645.7958 & 0.711 & 256.0 \\
\hline \multirow[t]{3}{*}{ 2000-4-12 } & 1646.6917 & 0.958 & 344.9 \\
\hline & 1646.7354 & 0.970 & 249.2 \\
\hline & 1646.7743 & 0.981 & 353.2 \\
\hline \multirow[t]{8}{*}{ 2000-10-21 } & 1838.7693 & 0.038 & 13.7 \\
\hline & 1838.8165 & 0.051 & 18.4 \\
\hline & 1838.8514 & 0.061 & 22.0 \\
\hline & 1838.9158 & 0.079 & 28.4 \\
\hline & 1838.9596 & 0.091 & 32.8 \\
\hline & 1839.0034 & 0.103 & 37.1 \\
\hline & 1839.0499 & 0.116 & 41.8 \\
\hline & 1839.0905 & 0.127 & 45.7 \\
\hline 2000-10-22 & 1839.7860 & 0.319 & 114.8 \\
\hline \multirow[t]{6}{*}{ 2000-10-23 } & 1840.7910 & 0.597 & 214.9 \\
\hline & 1840.8313 & 0.608 & 218.9 \\
\hline & 1840.9139 & 0.631 & 227.2 \\
\hline & 1840.9577 & 0.643 & 231.5 \\
\hline & 1841.0014 & 0.655 & 235.8 \\
\hline & 1841.0445 & 0.667 & 240.1 \\
\hline \multirow[t]{3}{*}{ 2000-10-24 } & 1841.7786 & 0.870 & 313.2 \\
\hline & 1841.8230 & 0.882 & 317.5 \\
\hline & 1841.9244 & 0.910 & 327.6 \\
\hline \multirow[t]{2}{*}{ 2000-10-25 } & 1842.8461 & 0.165 & 59.4 \\
\hline & 1842.8829 & 0.175 & 63.0 \\
\hline 2001-3-22 & 1990.7658 & 0.040 & 14.4 \\
\hline \multirow[t]{2}{*}{$2001-3-23$} & 1991.7595 & 0.315 & 113.4 \\
\hline & 1991.7921 & 0.324 & 116.6 \\
\hline \multirow[t]{5}{*}{ 2002-1-22 } & 2296.6475 & 0.568 & 204.5 \\
\hline & 2296.6755 & 0.576 & 207.4 \\
\hline & 2296.7035 & 0.582 & 209.5 \\
\hline & 2296.7335 & 0.589 & 212.3 \\
\hline & 2296.7606 & 0.600 & 215.9 \\
\hline \multirow[t]{3}{*}{ 2002-1-29 } & 2303.6545 & 0.505 & 181.8 \\
\hline & 2303.6830 & 0.512 & 184.3 \\
\hline & 2303.7110 & 0.520 & 187.2 \\
\hline \multirow[t]{4}{*}{ 2002-2-20 } & 2325.6614 & 0.585 & 210.6 \\
\hline & 2325.6817 & 0.592 & 213.1 \\
\hline & 2325.7206 & 0.604 & 217.4 \\
\hline & 2325.7674 & 0.615 & 221.3 \\
\hline \multirow[t]{3}{*}{$2002-4-8$} & 2372.6505 & 0.570 & 205.2 \\
\hline & 2372.6795 & 0.580 & 208.8 \\
\hline & 2372.7080 & 0.589 & 212.3 \\
\hline
\end{tabular}

Table 1. continued.

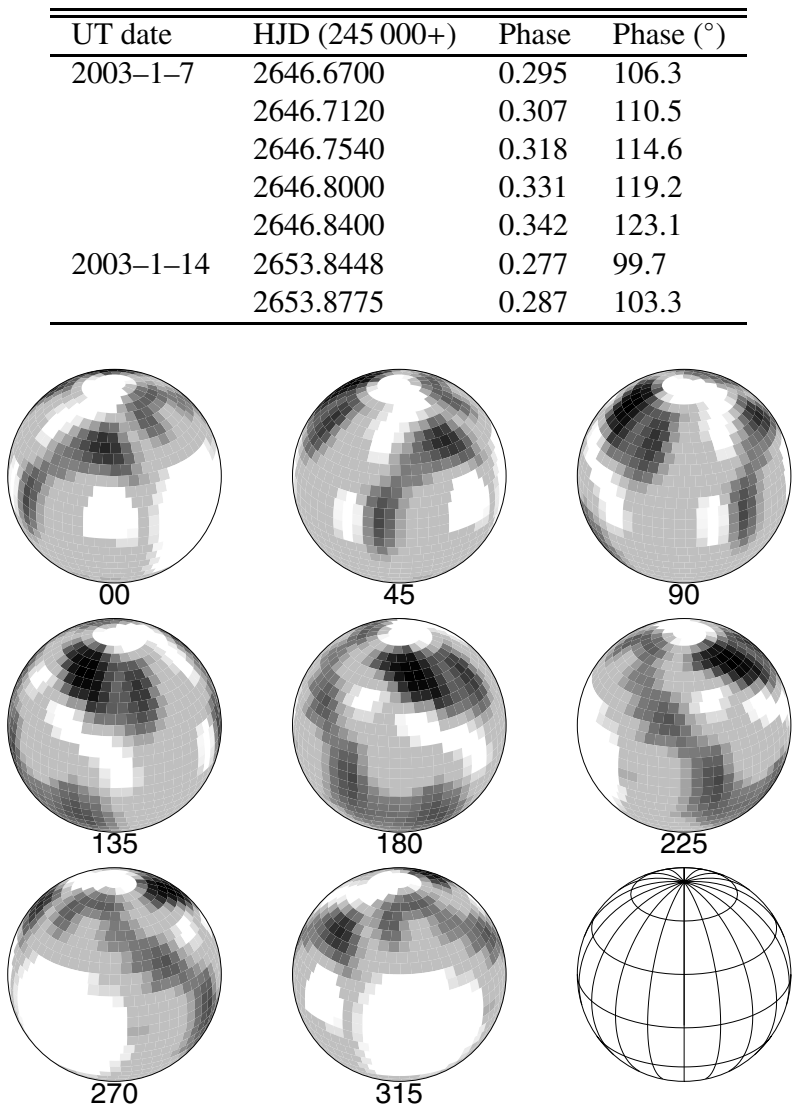

Fig. 1. The chromium image of theta Aur based on the same dataset as was used for plate 2 of Hatzes (1991a). This was generously provided by Hatzes to allow for more convenient comparison of the chromium and the oxygen image of this paper. The phases have been adjusted so that the images are displayed here on the basis of the ephemeris used in this paper and these images can be compared directly with Fig. 2. Note that the dark areas represent weaker chromium local equivalent width and the light regions represent stronger local line strength.

multicomponent stellar wind guided by the global magnetic field where some return fall to the surface produces the secondary patches.

Given the very interesting oxygen distribution on $\epsilon \mathrm{UMa}$, its potential implications for the mechanism responsible for the non-homogeneous abundance distribution of elements and its capacity to define the axis of symmetry that presumably is roughly coincident with the axis of magnetic symmetry, we suspected that a map of the oxygen distribution on another Ap star such as $\theta$ Aur would prove to be informative in the sense that it would support or confuse the apparent symmetry of Ap star oxygen distribution and the presence of any secondary abundance patches in comparison with $\epsilon \mathrm{UMa}$.

\section{The magnetic ephemeris and abundance symmetry}

The period of $\theta$ Aur has been fairly well established since Borra $\&$ Landstreet (1980) determined a period of 3.618 based on their sequence of magnetic observations. The zero point for 
their ephemeris was based on their estimate of the positive extremum of the magnetic variation. In a subsequent paper Adelman et al. (1984) adjusted the estimate of the period to 3.619 based on all combined magnetic and spectroscopic observations. Hatzes (1991a) further refined the period to 3.6187 and, based on his replot of the magnetic observations, adopted a slightly different zero point for the phases that represented his estimate of the time of positive magnetic extremum. The revised estimate of magnetic maximum did not seem significantly different from the choice of Borra \& Landstreet given the magnetic curve data. It was difficult to be precise about the peak of the curve of effective magnetic field. In this paper the zero point of Borra \& Landstreet has been combined with the period of Hatzes. The phases referred to in this paper are then calculated from:

$\mathrm{HJD}=2442766.55+3.6187 \times E$.

The zero point of Hatzes' (1991a) ephemeris occurs at phase 0.935 of the above ephemeris.

To put the observations of the chromium distribution on $\theta$ Aur from Hatzes' paper (1991a) into the context of this new ephemeris, we note that he observes a spot of enhanced chromium abundance at the equator and that spot extends in latitude to the lower limit of visibility (see Fig. 1 which is from the data of plate 2 in Hatzes 1991a). The spot is centred near phase 0.88 (roughly $320^{\circ}$ ) on Hatzes'original map and this location would translate to approximately phase $0.82\left(295^{\circ}\right)$ for our ephemeris and for the revised phases shown in Fig. 1. In either event the meridian crossing of this spot slightly preceeds the estimates of when maximum positive effective magnetic field occurs based on the observations of Borra and Landstreet (1980).

Given the uncertainty in identifying the occasion of the positive extremum in the observations of the effective magnetic field and given the symmetry evident in the chromium map of Hatzes, it seems that the spot of strong chromium abundance in his map is probably the location of the positive magnetic pole on the surface of $\theta$ Aur. If that is the case, we propose that the phase representing the time of crossing of the positive magnetic pole across the stellar meridian is at about phase 0.82 $\left(295^{\circ}\right)$ using our ephemeris. If it is not the case, the symmetry of the surface abundance distribution may be displaced from the magnetic orientation.

In a paper based on observations of the $V$ and $y$ light variations of $\theta$ Aur, Adelman (1997) finds essentially the same period we are using here but needs an adjustment in the zero point to bring the ephemeris into phase with the maxima of the light variation. His ephemeris is:

$\mathrm{HJD}=2446337.465+3.6188 \pm 0.0001 \times E$.

If we calculate the phase of the light curve maximum from the epoch of Adelman's ephemeris using our period we get a phase for maximum light of $286^{\circ}$. Using Adelman's period and the same calculation gives a nearly identical phase of $276^{\circ}$ for the light maximum. The light curve maximum seems to correspond reasonably well with the proposed time of the crossing of the centre of the $\mathrm{Cr}$ abundance spot we associate with the positive magnetic pole through the stellar meridian.
On the other side of the star from the spot of enhanced chromium that we take to be the approximate location of the positive magnetic pole is a region of depleted chromium that Hatzes notes as being at phase 0.50 (about $0.43=155^{\circ}$ with our ephemeris and as shown in Fig. 1) and that is centred at stellar latitude $55^{\circ}$. This spot would appear to be consistent with the approximate location of the negative magnetic pole. Between these two spots there is a belt of depleted chromium that, on one side of the star, crosses the line of sight at about Hatzes' phase 0.75 (roughly $240^{\circ}$ as shown in Fig. 1). In the case of the chromium map of $\epsilon$ UMa (Hatzes 1991b), a belt of depleted chromium abundance clearly outlines the magnetic equator of a simple (probably dipole) global magnetic field. In the case of the belt of depleted chromium in the map of $\theta$ Aur we suspect that, by analogy with $\epsilon \mathrm{UMa}$, the belt traces out the magnetic equator of a globally symmetric magnetic field. If we concentrate on the circle of depleted chromium around the apparent magnetic equator of $\theta$ Aur instead of the depleted spot apparently marking the negative magnetic pole, we would identify the axis of symmetry for the magnetic equator as coming to the meridian of the stellar disk a little earlier than the central part of the depleted spot representing the negative magnetic pole. This axis of symmetry would arrive at roughly phase 0.40 using Hatzes' ephemeris (or around phase $0.33=137^{\circ}$ with the ephemeris used here and as the Cr map of Hatzes is shown in Fig. 1). Based on the chromium maps of Hatzes (1991a), and continuing with the analogy to $\epsilon \mathrm{UMa}$, we would suspect that a point in the depleted region at roughly phase $140^{\circ}$ and latitude $+55^{\circ}$ is the approximate location of the axis of symmetry of the chromium distribution and the approximate location of the negative magnetic pole. Based on that assumption and using the ephemeris we have adopted for this paper, we would expect that we should find the axis of symmetry of the abundance pattern in the oxygen map (the point that we propose represents the approximate location of the negative pole) to be near longitude $135^{\circ}$ (phase 0.38 ) and latitude $+55^{\circ}$ (i.e. in the hemisphere of the visible pole). The positive pole should be near longitude $295^{\circ}$ and latitude $-55^{\circ}$.

The above discussion is important to the interpretation of the oxygen distribution reported below.

\section{The observations}

Sixty-five spectroscopic observations of $\theta$ Aur with a total exposure time of $40 \mathrm{~min}$ each were made over a period of almost three years from April 2000 to January 2003. The observations were made with the $1.2 \mathrm{~m}$ telescope of the Dominion Astrophysical Observatory using the 9681 camera that gives a dispersion of $5.0 \AA \mathrm{mm}^{-1}$ at a resolution of about 55000 at $7775 \AA$. The detector was a CCD chip of $4 \mathrm{k}$ pixels length. To ease removal of cosmic ray blemishes, each 40 min exposure is actually a combination of four $10 \mathrm{~min}$ observations. The optimal extraction of each one-dimensional spectrum was performed with MIDAS and the resultant $S / N$ in the continuum for each of the extracted spectra was fairly uniformly near 500 . Table 1 is a tabulation of the dates and phases of the observations where the phases are based on the ephemeris given in the previous section. 


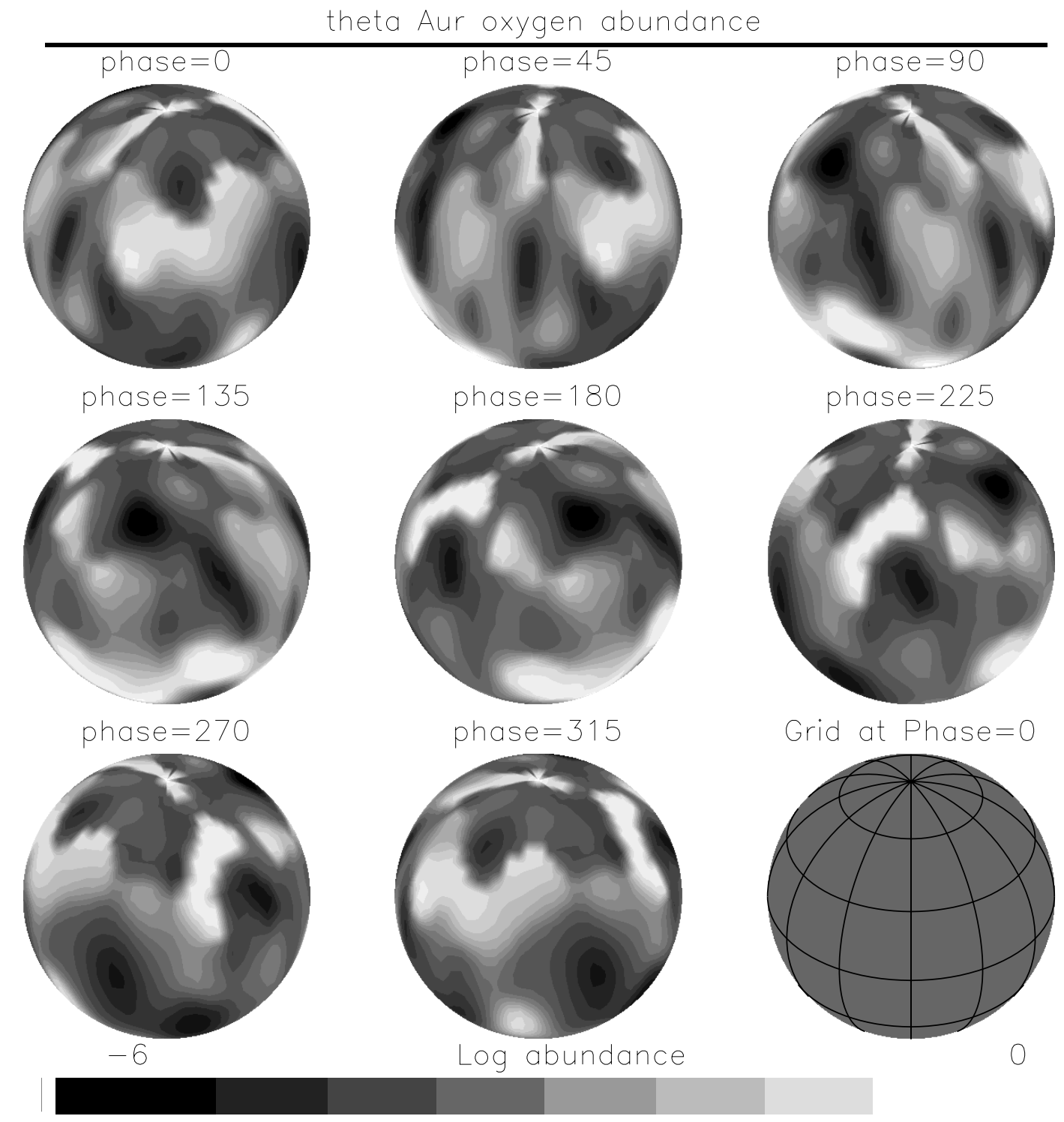

Fig. 2. Eight phases of the abundance map of the oxygen distribution over the surface of $\theta$ Aur. The upper left image is phase 0.0 with successive phases at intervals of 0.125 .

\section{The Doppler image}

A Doppler image of the abundance distribution of oxygen over the surface of $\theta$ Aur was produced from the spectroscopic observations using the same code used for the paper on $\epsilon \mathrm{UMa}$ (Rice et al. 1997). In choosing the appropriate model atmospheric parameters for use with the Doppler imaging code, the comprehensive papers by Adelman et al. (1984) and by van Rensbergen et al. (1984) were relied upon. These authors used for the general properties of the star the values $T_{\text {eff }}=$ $10450 \mathrm{~K}$ and $\log g=3.30$. For their atmosphere they used a ten times solar abundance model and they concluded that the turbulent velocities for $\theta$ Aur were very low. The estimate for the radius of $\theta$ Aur was that it was between $3.8 R_{\odot}$ and $7.1 R_{\odot}$.

In the earlier papers on Doppler images of $\theta$ Aur by Rice $\&$ Wehlau $(1990,1991)$, the values used for $v \sin i$ and $i$ were respectively $56.7 \mathrm{~km} \mathrm{~s}^{-1}$ and $50^{\circ}$. These maps suggested that the axis of symmetry that would correspond to the positive magnetic extremum for the distribution of iron, chromium and silicon was in the range of $50^{\circ}$ to $60^{\circ}$ from the visible rotational pole but different symmetry patterns are hypothesized. Hatzes (1991a) used $v \sin i$ and $i$ of respectively $53 \pm 2 \mathrm{~km} \mathrm{~s}^{-1}$ and $65^{\circ}$ for his Doppler image of the chromium distribution and he deduced a considerably different axis of symmetry for the chromium distribution than that of Rice \& Wehlau. In Hatzes work the axis of symmetry for the chromium distribution that would correspond to the positive magnetic extremum was at about $130^{\circ}$ to the visible rotational pole.

For the Doppler image of this paper the parameters given in Table 2 were adopted. The values shown for the atmospheric conditions were chosen to be consistent with Adelman et al. (1984) and van Rensbergen et al. (1984) as discussed above. The values adopted for $v \sin i$ and $i$ were evaluated independently here by varying these parameters over a reasonable range and choosing values that gave a fit to the spectroscopic observations of $\theta$ Aur that produced the minimum of error. 

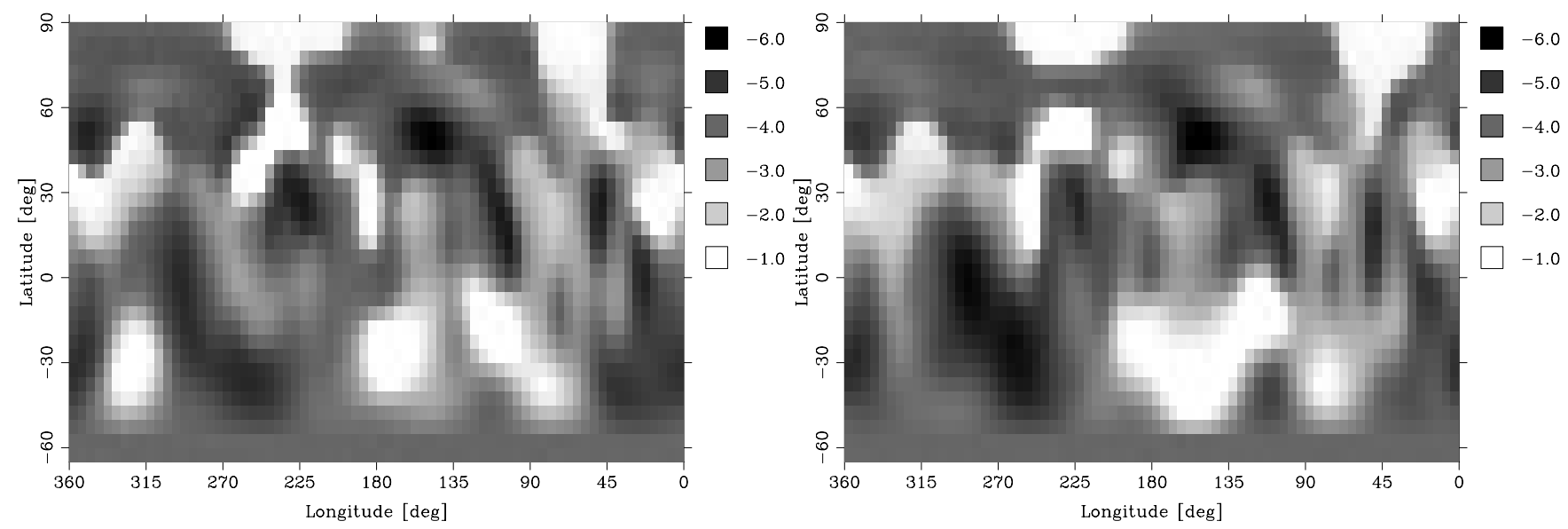

Fig. 3. Two Mercator style projections of the oxygen map of $\theta$ Aur, each based on half of the line profiles. The figure on the left is made from every other line profile listed in Table 1 beginning with the first (the odd profiles) and the figure on the right is made from every other line profile observation listed in Table 1 starting with the second profile (the even profile set). The two images represent images formed from two completely separate data sets. These alternative projections are provided for convenience in locating features by latitude and longitude and also to provide visual evidence of the errors that can be expected in Fig. 2.

The $v \sin i$ and $i$ of Table 2 are within the range already established by the previous work.

As has been shown in test papers (Piskunov \& Rice 1993; Rice et. al. 1989; Unruh \& Cameron 1995; Rice \& Strassmeier 2000), the final images in Doppler imaging are not very sensitive to variations in $i$ of the order of 10 degrees. The effect of errors in $v \sin i$ depends on the $v \sin i$ of the star in question but errors of greater than 0.5 to $1.0 \mathrm{~km} \mathrm{~s}^{-1}$ become significant on the same dataset. Comparisons between images recovered from different datasets using different $v \sin i$ values depends on the relative values of the resolution and the accuracy of the allowance for the instrumental profile and also the comparison between the values used for micro and macroturbulence. As shown in Rice \& Strassmeier (2000), even when numerous of these gross parameters have errors, the image is quite stable and the effect is on gross properties of the image such as equator to pole abundance or temperature. For a review of Doppler Imaging and some comparisons of Doppler Imaging by different authors working on the same star see Rice (2002).

A common strategy for evaluating the effect on the image of more detailed errors within a dataset of line profiles is to produce images from divided datasets. The usual division is to compare an image from the odd spectra observed with the image from the even spectra. The individual images may lose some detail because of the reduced input but a comparison of the variation of the detail in the images caused by stray errors in single spectra can be made. Here we present that comparison in Fig. 3. The two images shown in this figure represent images from two completely different sets of line profiles, the odd spectra from Table 1 and the even spectra. Each set of line profiles is only half as large as the original set.

The map computed for the oxygen distribution of $\theta$ Aur is shown in spherical projection in Fig. 2 and the Mercator projections of the same map are shown in Fig. 3. The reason for showing both versions is so that the spherical projection of Fig. 2 can be directly compared with Fig. 1, which is a spherical projection of the chromium image of $\theta$ Aur, while the Mercator
Table 2. Adopted parameters for Doppler imaging of $\theta$ Aur.

\begin{tabular}{ll}
\hline \hline Parameter & Adopted Value \\
\hline$T_{\text {eff }}$ & $10500 \mathrm{~K}$ \\
$\log g$ & 3.25 \\
$v \sin i$ & $52.0 \mathrm{~km} \mathrm{~s}^{-1}$ \\
Inclination $i$ & $54^{\circ}$ \\
Rotation period & 3.6187 days \\
Micro turbulence $\xi$ & $1.0 \mathrm{~km} \mathrm{~s}^{-1}$ \\
Macro turbulence & $0.0 \mathrm{~km} \mathrm{~s}^{-1}$ \\
Radius & 4.3 solar \\
\hline
\end{tabular}

style projections of Fig. 3 allow for easier identification of specific features by coordinate. To make it more convenient for the reader to make a comparison between his chromium map and the oxygen map presented here, Hatzes has generously provided us with the new Fig. 1 that is based on the same data as was used for his Plate 2 (Hatzes 1991a).

The fit of the forward calculation of the oxygen line triplet to the observed spectra on a phase-by-phase basis is shown in Fig. 4.

\section{Comparison with $\epsilon$ UMa and with the chromium map of Hatzes}

A direct comparison of the oxygen map of $\theta$ Aur in Fig. 2 with that of the oxygen map of $\epsilon$ UMa in Fig. 5 shows that $\theta$ Aur does not have the simple, single belt of normal oxygen abundance with extreme depletion of oxygen elsewhere that $\epsilon \mathrm{UMa}$ has. Only by referring to the discussion about the ephemeris in the second section of this paper is the more complex pattern for $\theta$ Aur evident. There we indicated that previous work has shown that the negative magnetic pole and the axis of symmetry of the chromium abundance is near longitude (or phase) $135^{\circ}$ and latitude $55^{\circ}$. In Fig. 2 we see that there is a double spot of pronounced depletion of oxygen at this location, and this depleted region is surrounded by a network of roughly solar 

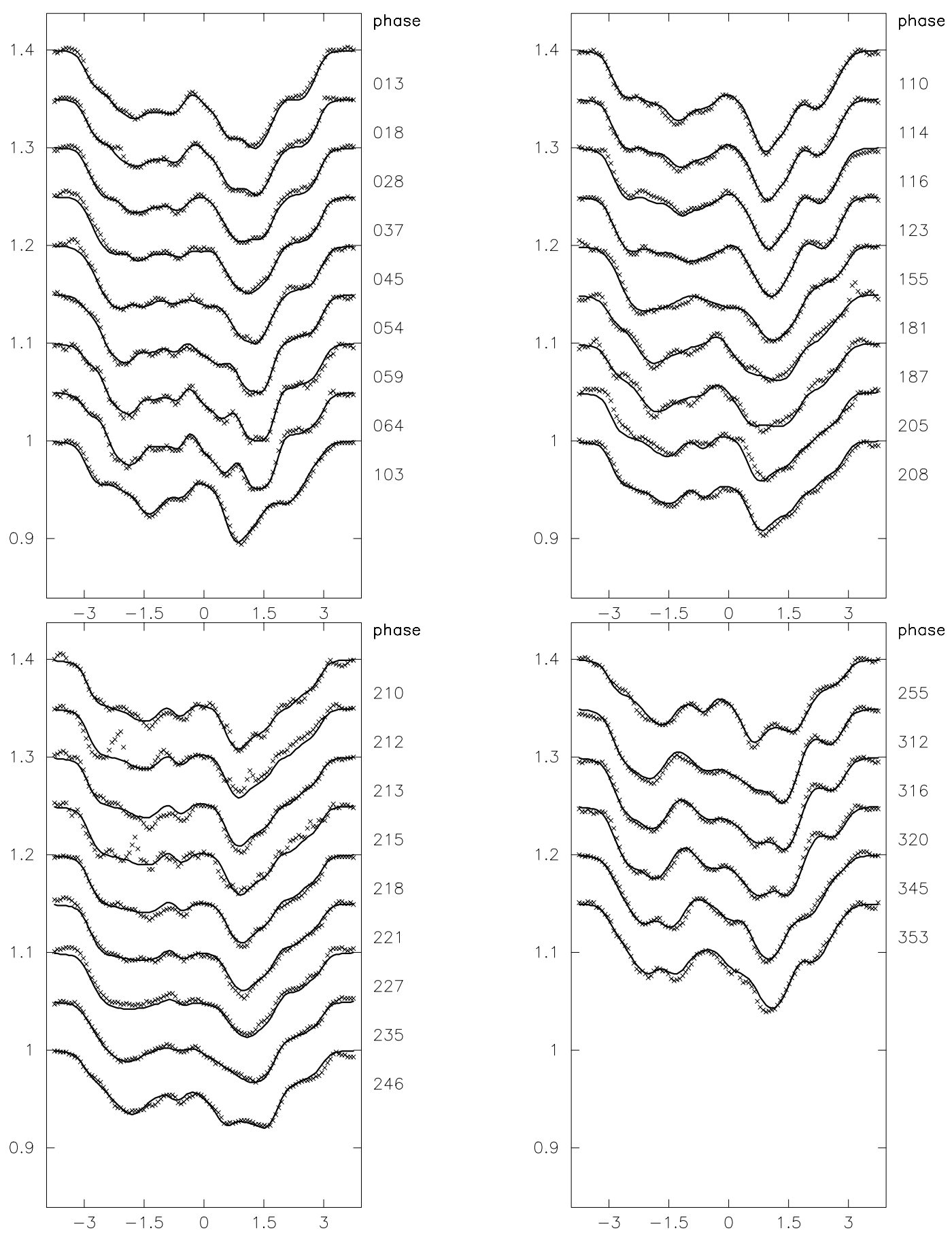

Fig. 4. Fit of predicted O I $\lambda 7775$ line profiles based on the Doppler image to the observed line profiles at half of the observed phases. Only every other spectrum and its fit is shown to allow for more detail in the display of the individual spectra. The spikes in the data at phases 212 and 215 have been removed from the Doppler Image reconstruction by having a weighting of zero applied to those data points.

oxygen abundance. Further, at roughly phase $270^{\circ}$ to $300^{\circ}$ and latitude $-30^{\circ}$ (near the limit of visibility) we see another zone of very depleted oxygen abundance where we would expect the positive magnetic pole. Both of these depletion zones are elongated rather than circular in shape (in fact, as noted above, the zone at phase $135^{\circ}$ is apparently significantly doubled). The depleted zone near the positive pole is also apparently surrounded by some irregular spots of roughly solar abundance but less apparently so than the depleted double zone at phase $135^{\circ}$. Generally, the region along the equator between these two centres of symmetry appears moderately depleted (i.e. at about abundance $[\mathrm{O} / \mathrm{H}]$ of less than -3 to as low as -4 when the solar oxygen abundance is about -3.1 ). The stark violation of the symmetry described here is the large spot of solar to slightly over solar abundance centred near phase (i.e. longitude) $0^{\circ}$ and latitude $+30^{\circ}$.

Given that Doppler Imaging is, by its nature, less reliable in the regions near the limit of visiblity in the hemisphere of the invisible pole, the two mercator images of the oxygen abundance map for $\theta$ Aur shown in Fig. 3 will allow the 


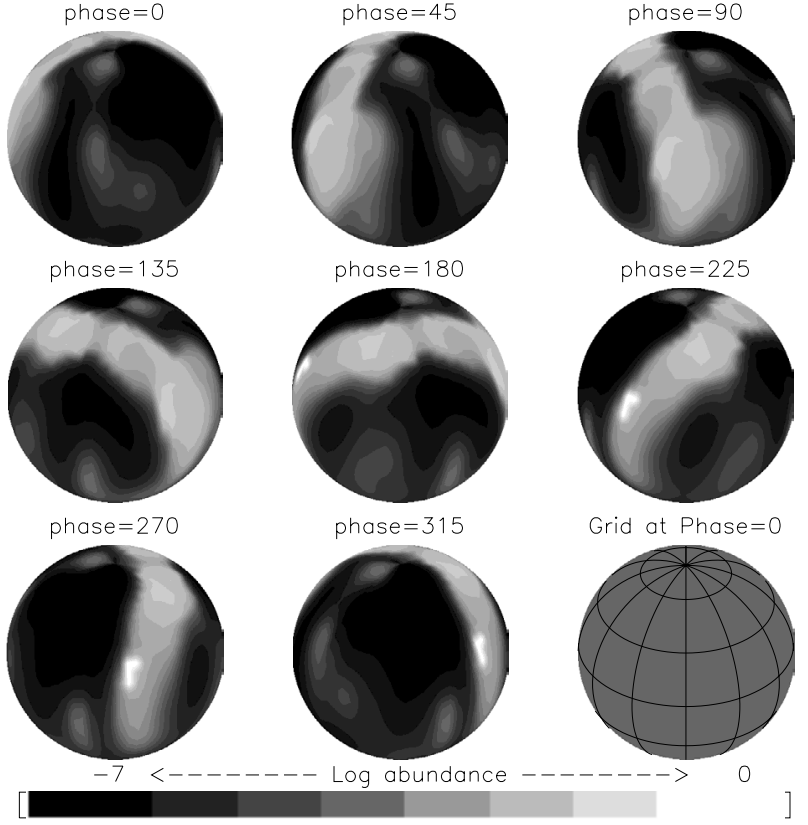

Fig. 5. Eight phases of the abundance map of the oxygen distribution over the surface of $\epsilon$ UMa from Rice et al. (1997). This old image is included here for convenience in making the comparison with the $\theta$ Aur map. You will note here that for $\epsilon$ UMa there is just one clear belt of normal oxygen abundance at roughly the magnetic equator of the star and that oxygen is depleted by many orders of magnitude at the magnetic poles.

reader to judge the reliability of the features we have discussed by directly comparing the two images formed from separate datasets.

If we calculate the phase of the light curve maximum from the epoch of Adelman's (1997) ephemeris using our period we get a phase for maximum light of $286^{\circ}$ as mentioned earlier. It appears that the phase of the maximum of the light curve is identical with the phase of the passage across the stellar meridian of the area of depleted oxygen in the lower hemisphere that we have identified with the positive magnetic pole, as also with the strong chromium spot in Hatzes' (1991a) map. Presuming the pattern above represents, in general, two spots of depleted oxygen near the magnetic poles, with each surrounded by irregular belts of approximately solar abundance with radii of about $45^{\circ}$ to $50^{\circ}$ and a zone around the magnetic equator of slighly below solar abundance, then this represents a significant deviation from the simple pattern of $\epsilon$ UMa. The pattern for $\theta$ Aur seems to suggest there is a significant quadruple component to the global magnetic field. We ignore the anomalous spot at phase $0^{\circ}$ and latitude $+30^{\circ}$ for now.

Next we compare the oxygen pattern with that of the chromium abundance distribution shown in plate 2 of Hatzes (1991a) and Fig. 1 here. Recall that for $\epsilon$ UMa the chromium map of Hatzes (1991b) was an almost perfect negative of the oxygen map we found in Rice et al. (1997). Where we found normal oxygen in $\epsilon$ UMa, Hatzes has a belt of depleted chromium and at the large regions of depleted oxygen in the $\epsilon$ UMa map, the chromium was of excess abundance compared with solar.
The comparison of oxygen and chromium for $\theta$ Aur is almost direct between our Fig. 2 and Hatzes' (1991a) plate 2 (Fig. 1 here which shows spherical projections with phasing adjusted to approximately that used in this paper). The depleted, elongated spots of oxygen at the presumed negative magnetic pole that we observe here in the oxygen map corresponds to an elongated region of depleted chromium, in contrast to the oxygen vs. chromium behaviour observed in $\epsilon$ UMa where depleted regions of oxygen correspond to enhanced chromium. In the chromium map the region of depleted abundance at the negative pole is surrounded by a pattern of enhanced chromium abundance that is quite similar in appearence to the pattern of solar oxygen abundence seen around the same pole here.

Just to complicate matters, at the presumed location of the positive pole at longitude $295^{\circ}$ and latitude $-55^{\circ}$, we see a feature of strong enhanced chromium abundance matching the position of an elongated region of depleted of oxygen in $\theta$ Aur. This is consistent with the behaviour in $\epsilon$ UMa. Around the presumed magnetic equator in Hatzes' (1991a) chromium map we see a belt of much weaker abundance that matches the slightly below solar abundance belt around the magnetic equator we see here. To illustrate the resemblence of the belts of lower abundance in the two images, the reader can compare the images shown at phase $270^{\circ}$ in Hatzes' plate 2 (Fig. 1 here) which has a remarkable resemblance to the image we show at phase $270^{\circ}$ in our Fig. 2. The only significant contrast between the two images is, as mentioned above, the large spot of excess abundance for chromium at the positive pole in Hatzes' image.

So far we have assumed that the symmetry of the oxygen and chromium maps better defines the locations of the magnetic poles and the phase when the effective magnetic field should be seen at extremum than the estimates of Borra \& Landstreet (1980) or Hatzes (1991a) based on the plots of the observations of the effective field. If this assumption is wrong and the aformentioned estimates of the peak in the observations of effective field are fairly accurate, we may have an indication that the field geometry is sufficiently complex that the time of maximum (or minimum) effective field does not coincide with the occasion when the axis of symmetry of the abundance distribution driven by the surface magnetic field is on the central meridian of the stellar disk. The maximum of the light curve does correspond quite closely with the time of passage of the axis of symmetry of the abundance distributions across the central meridian.

\section{Conclusions}

1. We find that $\theta$ Aur has a double elongated and irregular ring structure of normal solar abundance surrounding the highly depleted oxygen regions that seem to correspond to the magnetic poles. This is in contrast with $\epsilon$ UMa where a simple single band of roughly normal solar oxygen abundance was found at the equivalent location to the magnetic equator. We presumed, in the case of $\epsilon$ UMa that normal solar abundance occurred where the field lines were most horizontal and that the highly depleted oxygen regions corresponded to locations of vertical magnetic field lines. A reasonable assumption might be that the global structure of 
the magnetic field for $\theta$ Aur has a strong quadrupole component in contrast to $\epsilon \mathrm{UMa}$ where we have presumed that the very weak global field is predominantly a dipole configuration.

2. In comparing the chromium maps of $\epsilon$ UMa and $\theta$ Aur by Hatzes (1991a) we see that whereas the chromium map of $\epsilon$ UMa imitates the oxygen in the sense of having a belt of minimum chromium around the magnetic equator to match the oxygen belt at the magnetic equator, in $\theta$ Aur the chromium appears to be depleted at the negative pole where the oxygen is weak and enhanced at the apparent location of the positive pole where the oxygen is also weak. There is in both maps a tendency for the region of the magnetic equator to exhibit lower abundance than the peaks of abundance that appear in the two irregular bands surrounding the apparent magnetic poles.

3. The irregular nature of the band of solar to slightly above solar oxygen abundance that surrounds the two depleted spots that seem to represent the location of the negative pole have a pattern that is very similar to the spots of chromium enhancement in Hatzes' (1991a) map for $\theta$ Aur. Similarly, the anomalous spot (the one that seems to break the symmetry) at longitude near $0^{\circ}$ and latitude $+30^{\circ}$ has a counterpart in Hatzes map of chromium abundance, although seen in his map slightly closer to the equator. These irregularities appear to be quite real and not artifacts. Do these apparent departures from symmetry about the presumed magnetic axis suggest the field is more complicated even than simply a superposition of quadrupole and dipole components? If we refer to the Stokes $I$ and $V$ profiles of the spectrum lines as shown in Wade et al. (2000) we see a complex pattern of phased behaviour that might support the suggestion of a more complex global field structure.

4. In characterizing the features of the oxygen map here and the chromium map of Hatzes (1991a) in terms of the poles for the magnetic field we have concluded that the adjustment to the time of the apparent maximum of the effective magnetic field from the data of Borra \& Landstreet (1980) that was made by Hatzes comes closer to the occasions of meridian crossing of the presumed magnetic poles than the original ephemeris epoch adopted here. The time of the peak positive effective magnetic field assumed by Hatzes, as mentioned earlier, would be at phase $0.935=337^{\circ}$ using our ephemeris. The axis of symmetry that appears in our abundance map nearest the positive magnetic pole appears to be at roughly longitude $300^{\circ}$ and the axis of symmetry nearest the negative magnetic pole at roughly $135^{\circ}$. If the estimates of the occasion of the extrema of the curves of effective magnetic field are correct, the axis of symmetry of the abundance pattern generated by the magnetic field is apparently offset from the axis of the magnetic field itself. This would most likely signal a complex and possibly non-symmetric magnetic field. Note that the zero epoch of Adelman's (1997) ephemeris from the light curve appears to fit the meridian passage of the axis of symmetry of the abundance patterns of both the Doppler image of this paper and the chromium image of Hatzes (1991a).

5. The measured positive extremum of the effective magnetic field from Borra \& Landstreet (1980) is much stronger than the negative extremum. Since the positive pole, deduced from the abundance symmetry, appears to be at the extreme limit of visibility and the negative pole appears to cross through the centre of the visible disk of the star we must conclude that the surface strength of the field at the positive pole is much greater than that at the negative pole. This is likely a consequence of a superposition of quadrupole and dipole components of the global field.

Acknowledgements. The authors wish to thank the director of the Dominion Astrophysical Observatory for a generous allocation of observing time. This project was supported by a grant to J. B. Rice through the Natural Sciences and Engineering Research Council of Canada.

\section{References}

Adelman, S. 1997, PASP, 109, 9

Adelman, S., Hensberge, H., \& van Rensbergen, W. 1984, A\&AS, 57, 121

Borra, E. F., \& Landstreet, J. D. 1980, ApJS, 42, 421

Gonzalez, J.-F., \& Artru, M.-C. 1994, A\&A, 289, 209

Hatzes, A. 1991a, MNRAS, 248, 487

Hatzes, A. 1991b, MNRAS, 253, 89

Hiltner, W. A., \& Morgan, W. W. 1944, ApJ, 99, 318

Holmgren, D. E., \& Rice, J. B. 2000, A\&A, 364, 660

Khokhlova, V. L., Rice, J. B., \& Wehlau, W. H. 1986, ApJ, 307, 768

Piskunov, N. E., \& Rice, J. B. 1993, PASP, 105, 1415

Rice, J. B. 2002, AN, 313, 220

Rice, J. B., \& Strassmeier, K. G. 2000, A\&A, 147, 151

Rice, J. B., \& Wehlau, W. H. 1991, A\&A, 246, 195

Rice, J. B., \& Wehlau, W. H. 1990, A\&A, 233, 503

Rice, J. B., Wehlau, W. H., Khokhlova, V. L. 1989, A\&A, 208, 179

Rice, J. B., Wehlau, W. H., \& Holmgren, D. E. 1997, A\&A, 326, 988

Unruh, Y. C., \& Collier Cameron, A. 1995, MNRAS, 273, 1

van Rensbergen, W., Hensberge, H., \& Adelman, S. 1984, A\&A, 136, 31

Wade, G., Donati, J.-F., Landstreet, J., \& Shorlin, S. 2000, MNRAS, 313,823 